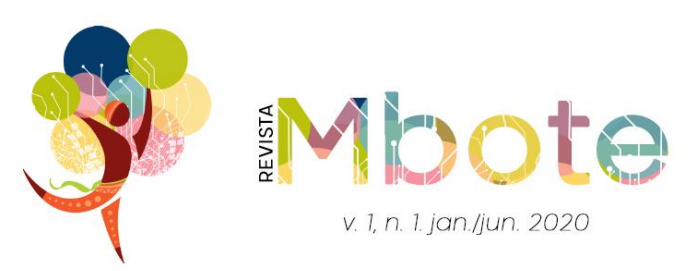

\title{
EQUIDADE, GÊNERO E DIREITOS HUMANOS: DESAFIOS EDUCACIONAIS NA AMÉRICA LATINA E O CARIBE ${ }^{1}$
}

\author{
Javier COLLADO-RUANO²; Cufuna Delsa SILVA-AMINO³
}

\begin{abstract}
Resumo: Este artigo tem o objetivo de refletir criticamente sobre a educação para a equidade de gênero na América Latina. Uma metodologia analítica e exploratória é utilizada para descrever a inequidade nas áreas rurais, onde existe maior índice de pobreza. Também se descreve a relação entre o empoderamento das mulheres com os Direitos Humanos e com o desenvolvimento sustentável. Então, percebe-se a necessidade de criar mais políticas públicas, programas e estratégias de responsabilidade compartilhada entre os diferentes agentes socioeducativos. Em suma, conclui-se que a implementação de práticas pedagógicas com foco na equidade de gênero contribui para erradicar e reduzir a pobreza de forma marcante.
\end{abstract}

Palavras-chave: Educação. Equidade de gênero. Direitos Humanos. Pobreza.

\section{EQUIDAD, GÉNERO Y DERECHOS HUMANOS: RETOS EDUCATIVOS EN AMÉRICA LATINA Y EL CARIBE}

Resumen: Este trabajo tiene el objetivo de reflexionar críticamente sobre la educación en equidad de género en América Latina. Se utiliza una metodología analítica y exploratoria que describe la inequidad de las zonas rurales, donde existe un mayor índice de pobreza. También se describe la relación entre el empoderamiento de las mujeres con los Derechos Humanos y con el desarrollo sostenible. Como resultado, se discierne que es necesario crear más políticas públicas, programas y estrategias de responsabilidad compartida entre los diferentes agentes socio-educativo. En suma, se concluye que la implementación de prácticas pedagógicas enfocadas en la equidad de género contribuyen a erradicar y disminuir la pobreza de forma notable.

Palabras clave: Educación. Equidad de género. Derechos Humanos. Pobreza.

\section{EQUITY, GENDER AND HUMAN RIGHTS: EDUCATIONAL CHALLENGES IN LATIN AMERICA AND THE CARIBBEAN}

\begin{abstract}
This paper has the objective to reflect critically on education in gender equality in Latin America. An analytical and exploratory methodology is used to describe the inequality of rural areas, where there is a higher poverty rate. It also describes the relationship between the empowerment of women with Human Rights and with sustainable development. As a result, it is discerned that it is necessary to create more

\footnotetext{
${ }^{1}$ Artigo publicado parcialmente em Andaluciaeduca.com

2 Professor de Filosofia da Educação da Universidade Nacional de Educação (UNAE) do Equador. Doutor em Difusão de Conhecimento pela Universidade Federal da Bahia (UFBA), e Doutor em Filosofia pela Universidade de Salamanca (Espanha). Mestre em Sociologia da Educação pela Universidade de Sevilha (Espanha). Graduação em História pela Universidade de Valencia (Espanha) e especialização em Relações Internacionais e Arqueologia pela Universidade degli Studi di Palermo (Itália). Membro do CIRET da França. Diretor de Inovação Educativa na Universidade Nacional de Educação (UNAE) (Javier de Loyola, Azuay, Ecuador). E-mail: javier.collado@unae.edu.ec, Website: www.javiercolladoruano.com

3 Pesquisadora da Universidade Nacional de Educação (UNAE) do Equador. Colaboradora de projetos de pesquisa educacional: TO INN; Educação e pobreza; A arte perdida da educação; Criatividade e inovação no currículo e pertence ao GRUPO TRENDS.
} 
public policies, programs and strategies of shared responsibility among the different socio-educational agents. In sum, it is concluded that the implementation of pedagogical practices focused on gender equality contribute to eradicate and reduce poverty in a remarkable way.

Keywords: Education. Gender equality. Human rights. Poverty.

\section{EDUCAÇÃO EM EQUIDADE DE GÊNERO PARA COMBATER A POBREZA}

No ano 2020, existem muitas iniciativas que têm como objetivo erradicar a pobreza e reduzir a fome no mundo. Os Objetivos de Desenvolvimento Sustentável (ODS) para 2030 procuram empoderar às mulheres e defender os seus direitos humanos, adotando 17 objetivos e 169 metas. Mas, o que se entende por pobreza desde uma perspectiva de gênero? Como medir a pobreza desde uma perspectiva de gênero? Como enfrentar a pobreza desde políticas públicas e programas educativos de equidade de gênero? Qual é o papel do pessoal docente para acabar com o machismo e o patriarcado histórico que existe de forma estrutural na América Latina e o Caribe? Quais são as críticas dos movimentos feministas emergentes?

Para responder a estas perguntas, é preciso entender a complexidade do mundo interconectado de hoje. Além disso, a pandemia da COVID-19 revelou a estreita relação entre saúde, educação e pobreza a nível global. De acordo com o Instituto Internacional de Educação Superior na América Latina e no Caribe (IESALC, 2020), as escolas e universidades tiveram que fechar devido à emergência sanitária do coronavírus: afetando 1,57 bilhões de estudantes em 191 países. Na região latinoamericana, 156 milhões de estudantes foram forçados a estudar em casa. Neste contexto, estima-se que cerca de 39\% desses estudantes vivem em áreas rurais, sem acesso à Internet ou à infraestrutura básica que lhes permitiria praticar a educação online, virtual ou à distância (UNESCO, 2020).

Quando falamos de educação em equidade de gênero para reduzir a pobreza, podemos ver que ela é um pilar fundamental construir a igualdade social (MÁRQUEZ, GUTIÉRREZ, GÓMEZ, 2017). Dentro deste contexto de emergência, o empoderamento de meninas, jovens e mulheres requer mudanças estruturais que levem a uma cultura de equidade. Evidentemente, este processo de transformação precisa do protagonismo da mulher como protagonista da mudança socioeconômica. 
Para Kabeer (1999), o empoderamento é um processo pessoal e coletivo, e a educação deve reforçar a autoconfiança, o desenvolvimento do pensamento crítico, o fortalecimento do grupo e a coesão, assim como as ações coletivas destinadas a quebrar as atuais relações de poder.

Para Rocha e Rocha $(2013,2014)$, alcançar um maior empoderamento das mulheres requer a promoção de mudanças nas políticas públicas, leis, instituições, relações de mercado e cultura. Desta forma, é possível obter um impacto direto na redução da pobreza e no cumprimento dos direitos humanos, bem como a plena cidadania das mulheres, com igualdade de oportunidades. Mudar as injustiças históricas e as relações de poder de gênero requer vontade política, consenso social e programas educacionais que aumentem a conscientização e sensibilização da sociedade. A ordem patriarcal hegemônica leva a uma distribuição mais injusta dos recursos e do poder do mundo. As consequências da inequidade para as mulheres são muito mais graves em termos de pobreza, em comparação com os homens, especialmente nas áreas rurais (SAGREDO, GALARZA, 2020).

No contexto rural, os direitos da mulher - civis, políticos, econômicos, sociais e culturais - são mais facilmente violados (BAKKER, 1994). A fim de mudar as condições de vida nas áreas rurais dos diferentes países da América Latina e do Caribe, é urgente fomentar políticas públicas de educação em equidade de gênero (ARAGONÉS, ROSSER, GIL, 2020). Portanto, este artigo se justifica em afirmar e evidenciar que a educação em equidade de gênero é um instrumento necessário para a transformação social. Sem trabalhar a questão educativa da equidade de gênero no currículo, não é possível avançar em direção a uma cidadania democrática inclusiva que reconheça os direitos humanos, nem será possível proporcionar as ferramentas para combater a pobreza no continente latino-americano (DEERE, ALVARADO, TWYMAN, 2018).

\section{EDUCAÇÃO EM EQÜIDADE PARA ACABAR COM A BRECHA DE GÊNERO}

O que é equidade de gênero? De acordo com Srinivasan e Rodríguez (2016), a equidade de gênero é baseada na observação das desigualdades entre homens e mulheres, e levanta a necessidade de justiça na distribuição de recursos e poder entre 
as pessoas. De acordo com García (2008), conseguir a equidade de gênero é essencial para eliminar todas as formas de discriminação contra a mulher, promover a autonomia e assegurar seu pleno desenvolvimento em todas as áreas da vida social, econômica e política, a fim de garantir o exercício da cidadania e o gozo dos direitos humanos e das liberdades fundamentais em condições de igualdade com os homens. Se desejamos avançar em rumo a uma sociedade igualitária na América Latina e no Caribe, precisamos repensar as estratégias de integração de gênero na educação a fim de integrar todos os setores e organizações nacionais, regionais e internacionais.

Para conseguir isto, é preciso começar a implementar uma política de educação intercultural latino-americana que incorpore a diversidade de culturas, populações e relações sociais no reconhecimento de uma cidadania planetária justa e democrática, em favor da erradicação da pobreza (COLLADO, 2018). Neste sentido, a Declaração dos Direitos Humanos Universais atua como um padrão internacional. Como padrão supranacional e consensual, os direitos humanos constituem o marco de referência mais legítimo para analisar os problemas sociais e para sustentar propostas pedagógicas para lidar com eles. Esta abordagem pedagógica intercultural da equidade mostra que o desenvolvimento socioeconômico está intimamente ligado ao fim da pobreza, à equidade de gênero e à emancipação da mulher, especialmente nas áreas rurais e nas áreas peri-urbanas mais desfavorecidas (BENERÍA, 2003).

Desde um ponto de vista histórico, Angrisani, Lee e Meijer (2020) apresentam evidências científicas que mostram como as mulheres tiveram um nível de educação inferior ao dos homens, e que a brecha de inequidade vem diminuindo ao longo do tempo. Consequentemente, pode-se dizer que a educação é um elemento fundamental para reduzir a diferença de gênero na América Latina e no Caribe, uma vez que expande significativamente as oportunidades socioeconômicas. Portanto, a disparidade econômica, a desnutrição infantil, o acesso à educação primária e secundária e a inequidade de gênero são fatores limitantes para reduzir o nível de pobreza a curto, médio e longo prazo na região. Além disso, as altas taxas de gravidez entre meninas adolescentes impedem que elas continuem seus estudos (PAHO, 2016).

\section{A RELAÇÃO ENTRE POBREZA E GÊNERO NAS ÁREAS RURAIS DA AMÉRICA}




\section{LATINA E DO CARIBE}

A lacuna entre os jovens que têm acesso ao ensino primário e secundário nas áreas urbanas e rurais continuou aumentando nos últimos anos (CEPAL, 2017a). Assim, é necessário criar propostas de gestão da educação que focalizem a melhoria da qualidade dos serviços nas áreas rurais da América Latina e do Caribe. Uma desvantagem histórica é a falta de informação nestas áreas, uma vez que as mulheres das áreas urbanas têm maior acesso à informação sobre seus direitos e maiores oportunidades de estabelecer redes organizacionais para reivindicar e defender os seus direitos diante das agências governamentais. Porém, os movimentos urbanos têm poucas conexões com as áreas rurais e, portanto, os direitos alcançados não foram muito abrangentes para as mulheres mais pobres (ARRIAGADA, 1997).

Por outro lado, estudos da CEPAL (2017b) indicam que as mulheres das áreas rurais da América Latina têm as mais altas taxas de analfabetismo, mais crianças em média, níveis de educação mais baixos, pouca organização política e, em suma, uma carga de trabalho mais pesada nas esferas doméstica, comunitária e produtiva. Ao mesmo tempo, também devem lutar contra várias formas de violência, discriminação, misoginia e feminicídio, pois estão mais desprotegidas contra a violência doméstica e têm menos acesso a todos os tipos de serviços de apoio nas áreas rurais. Segundo Giosa e Rodríguez (2010), a falta de informação em línguas indígenas leva a pouca ou nenhuma participação em redes e organizações nacionais de movimentos de mulheres. Portanto, a pobreza tem um rosto de mulher nas áreas rurais da América Latina e do Caribe.

Para as Nações Unidas (2015), o período de 2000 a 2010 trouxe um crescimento econômico muito importante, especialmente na Argentina, Brasil e México. Este desenvolvimento diminuiu o número de pessoas pobres na região, embora ainda existem 216 milhões de pessoas (38\% da população) em risco de viver na pobreza. Por esta razão, é preciso aprofundar o debate sobre novas políticas públicas e objetivos regionais que permitam a criação de condições de segurança para as famílias rurais mais ameaçadas da região. Embora os esforços da sociedade civil, dos governos da região e das instituições internacionais têm melhorado os direitos das 
meninas a frequentar a escola, ainda há muitos desafios para conseguir melhores condições de trabalho para as mulheres e maior participação na tomada de decisões políticas. A conclusão é clara: a exclusão e a discriminação de gênero são acentuadas significativamente à medida que aumenta a pobreza em que vivem as mulheres (PRETO, 2019).

\section{DIREITOS HUMANOS E POBREZA: DESAFIOS PARA O DESENVOLVIMENTO}

De acordo com Saraví (2020), a América Latina e o Caribe são caracterizados como as regiões do mundo com pior distribuição de renda, razão pela qual se acumulam desvantagens e uma grande inequidade de gênero. Apesar da tendência de redução das disparidades de gênero nas últimas décadas, ainda há muito trabalho a ser feito para garantir que homens e mulheres tenham as mesmas liberdades, direitos e oportunidades para o desenvolvimento individual e coletivo (DE LA CRUZ, 2009).

Desde um ponto de vista histórico, a Declaração dos Direitos Humanos de 1948 foi marcada pelo contexto de crise civilizacional deixado pelos conflitos bélicos do século XX. Da mesma forma, os Direitos Humanos da Mulher constituíram um marco histórico para a construção da democracia, da justiça social, do progresso dos povos e da paz mundial (BERIK, RODGERS, ZAMMIT, 2008). Neste sentido, a Convenção sobre a Eliminação de todas as formas de Discriminação contra a Mulher de 1979, conhecida como CEDAW, marcou uma das maiores proezas na história para corroborar os direitos da mulher. Todos os países da América Latina e mais de 130 países do mundo também aderiram-se a ela, se comprometendo a tomar medidas que ampliassem, favorecessem e garantissem os direitos humanos das mulheres.

Algumas décadas depois, a IV Conferência Mundial realizada em Beijing em 1995 pela ONU abordou a situação de pobreza das meninas, violência contra as mulheres, exclusão econômica, direitos à saúde e educação. No ano 2000, as Nações Unidas aprovaram os Objetivos do Milênio (ODM) com uma agenda global para orientar as ações locais, nacionais e regionais para superar a pobreza e o desenvolvimento sustentável (COLLADO, 2017). Tais objetivos estabeleceram metas concretas para melhorar a situação da mulher. No campo da educação, eles se concentraram na 
universalização e na paridade entre os meninos e meninas nos níveis primário e secundário (GRIMALDO, CERVERA, 2016). Em 2015, os Estados Membros da ONU elaboraram os Objetivos de Desenvolvimento Sustentável (ODS) para enfrentar os desafios sócio-ecológicos da nossa contemporaneidade.

Muitas mulheres na América Latina e Caribe ainda não têm os mesmos direitos e oportunidades que os homens, especialmente nas áreas rurais. As funções sociais relacionadas à reprodução são derivadas da capacidade biológica do corpo feminino de procriar. Enquanto os homens assumem o espaço público para controlar áreas estratégicas como a economia e a política, as mulheres são relegadas ao espaço doméstico, para cuidar das crianças e das tarefas da vida diária. Esta situação gerou, desafortunadamente, um processo de inferiorização das funções biológicas e sociais da mulher, o que se traduz na desvalorização da mulher como cidadania, ou não é verdade que o trabalho fora de casa é valorizado e recompensado, enquanto o trabalho dentro de casa é invisível, desvalorizado e não remunerado?

Depois de analisar a autonomia econômica, física e decisória, o Observatório de Igualdade de Gênero para a América Latina e o Caribe estima que muitas mulheres são consideradas como cidadãs de segunda classe ou cidadãs da categoria mais baixa, e que os seus direitos estão sendo restringidos (CEPAL, 2017b). Esta mentalidade patriarcal e machista é perturbadora, e uma transformação social é necessária para fechar a brecha histórica de inequidade da região (FULLER, 1998). Por outro lado, desde um ponto de vista sócio-crítico, é preciso assinalar que os indicadores utilizados pelas instituições para estudar a exclusão de gênero somente declaram a equidade de gênero a partir de uma lógica heterossexual e binária (homem-mulher) (BARRIENTOS, 2018). Por esta razão, quase não há estudos que mostrem a situação de exclusão social e discriminação homofóbica, biofóbica, transfóbica e queerofóbica sofrida pelos gêneros mais vulneráveis (BUTLER, 2007).

\section{POLÍTICAS PÚBLICAS COM PERSPECTIVA DE GÊNERO}

As políticas públicas com perspectiva de gênero devem ser orientadas para mudar as estruturas sociais organizacionais, razão pela qual as estratégias de Gender 
Mainstreaming precisam de uma análise multidimensional que aborde os desafios e as oportunidades. Tais transformações partem do princípio de que as organizações assumem o empoderamento como um processo de mudança induzido em uma ampla variedade de dimensões sociais. Isto implica considerar uma série de fatores a médio prazo: vontade política e legitimidade da matéria, recursos econômicos, formação administrativa e operacional da equipe de execução, o marco normativo ou jurídico e, de fato, que seja sócio-culturalmente viável (MOMSEN, 2019).

As políticas públicas com perspectiva de gênero devem ser incorporadas em todos os níveis de educação, do pré-escolar ao primário, secundário e universitário, como é o caso do desenvolvimento sustentável (COLLADO, 2019). O eixo da integração da perspectiva de gênero deve contribuir para mudar as relações de poder desiguais que são institucionalizadas, para que as mulheres tenham igual acesso aos mesmos recursos, direitos, oportunidades e participação que os homens. Neste sentido, os países da região devem aplicar políticas públicas de 'inclusão da perspectiva de gênero' para lograr uma equidade efetiva dos Direitos Humanos das Mulheres. Este processo de transformação e desenvolvimento social implica uma política de educação pública que aborde a heterogeneidade das áreas urbanas, periurbanas e rurais através de uma análise setorial de planificação, execução e avaliação.

Mas o que significa tudo isso na prática? Em primeiro lugar, é preciso que exista vontade política por parte dos governos latino-americanos, já que eles precisam se comprometer a atribuir recursos financeiros e humanos suficientes. Estes recursos poderão ser controlados pelo Observatório de Igualdade de Gênero para a América Latina e o Caribe (2008). Em segundo lugar, a estratégia de empoderamento não deve se sobrepor à necessidade de ações específicas de desenvolvimento em favor das mulheres e, portanto, da atribuição de recursos concretos. É necessário ter em consideração que as políticas locais de equidade são criadas para resolver um problema específico resultante de uma determinada área com fenômenos diversos.

Em terceiro lugar, é importante que exista esclarecimento e consenso por parte de todos os atores responsáveis que participam da estratégia de Gender Mainstreaming de cada instituição latino-americana, nos níveis regional, nacional e local. Por isso, a 
existência de instituições e pessoas com treinamento especializado e responsabilidade pela promoção da equidade de gênero é fundamental para maximizar os esforços e servir de estímulo e ímpeto em outras áreas. Em quarto lugar, é necessário elaborar e implementar ações de educação em equidade de gênero que funcionem como um eixo transversal que atenda às políticas públicas de educação existentes. Esta abordagem transversal deve promover uma transformação na mentalidade da cidadania.

Em quinto e último lugar, é preciso elaborar ferramentas TIC para analisar e planificar adecuadamente, com o fim de proporcionar maior capacitação e conhecimento das estruturas e mecanismos institucionais para aqueles que implementam novas políticas públicas educativas que promovam a equidade de gênero. Isto requer investimento de recursos na divulgação de informações, dados desagregados e pesquisas que ajudem a identificar às inequidades de gênero. Também implica conhecer as razões da baixa representação das mulheres na vida política e pública, o que impede que elas tomem decisões para dar poder a uma cidadania crítica que exige seus direitos (NUSSBAUM, 2000).

Com estas diversas ações destinadas a melhorar as condições organizacionais, econômicas e produtivas, o objetivo das políticas públicas educativas com perspectiva de gênero ajudariam a diminuir e erradicar a pobreza. Mas isso implica uma profunda transformação na lógica organizacional das instituições. Evidentemente, o papel da profissão docente é essencial para conseguir o fim da desigualdade e da pobreza social, especialmente nas áreas rurais. Assim, o corpo docente precisa se atualizar através de cursos de formação pedagógica transdisciplinar que lhes permitam adaptar a equidade e a igualdade de gênero a seus contextos socioeducativos, com a finalidade de diminuir a pobreza na região (DRAVET et al., 2019). A juventude latinoamericana depende da capacitação dos docentes para transformar as desigualdades históricas estruturais na sua raiz, e por isto os governos devem garantir as condições de trabalho decentes para os professores e professoras.

Então, falar de Direitos Humanos Universais no século XXI significa gerar políticas públicas de equidade de gênero para construir uma cidadania plena, tendo às mulheres como pilares de uma civilização justa, democrática e sustentável. 
Efetivamente, o conceito de pobreza multidimensional considera o nível material das pessoas, assim como sua formação educacional, as suas oportunidades de emprego e a sua saúde socioambiental. É por isso que as políticas públicas de Gender Mainstreaming devem ser aplicadas com especial ênfase nas áreas rurais, com o propósito de responder às necessidades, interesses e exigências das mulheres nestes contextos. Sem dúvida, a emancipação e o empoderamento das mulheres terão um impacto positivo no desenvolvimento da região, contribuindo para a erradicação da pobreza.

\section{REFLEXÕES PARA ALCANÇAR A AGENDA 2030}

Além de pesquisar e analisar dados estatísticos, cientistas e acadêmicos têm uma grande responsabilidade ética para formular propostas que ajudem aos governos e a sociedade civil a (re)pensar as políticas públicas e as estratégias de cooperação regional. As reflexões e os argumentos desta pesquisa nos permitem propor o eixo transversal da equidade de gênero em todos os níveis educativos através de políticas públicas adaptadas a cada país da região. Embora temos que reconhecer as vozes críticas que qualificam a Agenda 2030 como colonial, monocultural e ocidentalista (HIDALGO et al., 2019), os ODS têm aberto uma porta para o diálogo internacional e para enfrentar os desafios socioambientais contemporâneos de uma forma conjunta e colaborativa.

Trabalhar pelos direitos humanos, pela equidade de gênero e pela erradicação da pobreza significa, por conseguinte, aprender a cooperar a partir da grande diversidade da cidadania. Por isso, as estratégias políticas para aplicar a abordagem de equidade de gênero devem ser sustentadas por abordagens multidimensionais que se apoiem na diversidade dos cidadãos como uma força de transformação. Portanto, é necessário que os docentes se envolvam na mudança social em todos os níveis, apoiando diferentes tipos de estratégias para melhorar a equidade de gênero nas escolas, institutos e universidades. Estas estratégias devem ser integradas, combinadas e aplicadas de acordo com o contexto socioeducativo. Sem dúvida, este eixo educacional de equidade de gênero deve continuar fora da sala de aula, por isso é necessário se conectar com a sociedade para que as famílias sejam corresponsáveis. 
Neste sentido, o corpo docente precisa ser capaz de pesquisar a fonte das desigualdades entre estudantes, com o objetivo de se desenvolver com as mesmas perspectivas e oportunidades futuras de acesso à universidade e ao mercado de trabalho. Portanto, o conceito de democracia deve ser ampliado para que os direitos específicos das mulheres sejam consagrados no marco legal e sejam tomadas ações positivas que considerem as diferenças biológicas e socialmente construídas. Para acabar com as desigualdades históricas, os docentes devem fazer uma leitura crítica do mundo que sirva para empoderar aos seus estudantes (FREIRE, 1997).

Assim, as políticas públicas educativas sobre equidade de gênero têm que ser integradas em toda a região, especialmente nas áreas rurais mais desfavorecidas. Esta abordagem tem que denunciar as injustiças entre os estudantes, assim como transformar os fenômenos de inequidade nas relações de poder. Consequentemente, é uma questão de se questionar tanto os processos internos dentro das organizações, como também os processos externos no nível social, onde todas as ações deverão ter estratégias que ajudem a prevenir e resolver a inequidade de gênero, a fim de contribuir para mudanças mais permanentes.

Portanto, os centros de educação rural da região devem ter como diretriz pedagógica o desenvolvimento de habilidades humanas que levem a um mundo mais justo, livre da pobreza, onde as oportunidades para crianças e jovens sejam equitativas. E qual seria o primeiro passo para alcançar isso? Sem dúvida, conseguir que o corpo docente das áreas rurais melhore a sua condição de trabalho, com o propósito de poder se concentrar no treinamento contínuo que lhes permita resolver situações de desigualdade dentro da sala de aula. O pensamento de Paulo Freire (1971) permanece vigente, considerando que os professores devem ensinar a partir de uma pedagogia crítica, criativa e participativa que promova a ação pessoal e coletiva para melhorar o complexo mundo de hoje.

\section{REFERÊNCIAS}

ANGRISANI, M., LEE, J., \& MEIJER, E. (2020). The gender gap in education and late-life cognition: Evidence from multiple countries and birth cohorts. The Journal of the Economics of Ageing, vol. 16, pp. 1-16. 
https://doi.org/10.1016/j.jeoa.2019.100232

ARAGONÉS, M., ROSSER, A., GIL, D. (2020). Coeducation and gender equality in education systems: A scoping review. Children and Youth Services Review, vol. 111, pp. 1-12. https://doi.org/10.1016/j.childyouth.2020.104837

ARRIAGADA, I. (1997). Realidades y mitos del trabajo femenino urbano en américa latina. Serie mujer y desarrollo, 21. Santiago de Chile: Cepal.

BARRIENTOS, J. (2018). "Educación y pleno respeto a la diversidad de género" Mensaje, vol. 67, no. 673, p. 38+. Gale OneFile: Informe Académico.

BAKKER, I. (1994). Engendering macro-economic policy reform in the era of global restructuring and adjustment. The strategic silence: gender and economic policy (pp. 1-29). London: zed books.

BENERÍA, L. (2003). Gender, development and globalization. New York: Routledge.

BERIK, G., RODGERS Y. Y ZAMMIT P. (2008). Social justices and gender equity: rethinking development strategies and macro-economic policies. London: Routledge.

BLACK, N. (2019). Social Feminism. London: Cornell University Press.

BUTLER, J. (2007). El género en disputa. Barcelona: Paidós.

CEPAL (2017a). Comisión Económica para América Latina y el Caribe, Brechas, ejes y desafíos en el vínculo entre lo social y lo productivo. Santiago: CEPAL.

CEPAL. (2017b). Planes de igualdad de género en América Latina y el Caribe. Mapas de ruta para el desarrollo. Santiago: CEPAL

COLLADO, J. (2017). O desenvolvimento sustentável na educação superior. Propostas biomiméticas e transdisciplinares. In: Revista lberoamericana de Educación, vol. 73, pp. 203-224.

COLLADO, J. (2018). Um olhar transdisciplinar e biomimético à Educação para a Cidadania Planetária e aos Objetivos de Desenvolvimento Sustentável. Curriculo sem fronteiras, vol. 18, №2, pp. 500-529.

COLLADO, J. (2019). Reflexões filosóficas sobre a Educação para o Desenvolvimento Sustentável: uma abordagem complexa, transdisciplinar e biomimética. Foro de Educación vol. 17, noำ26, pp. 135-152.

DE LA CRUZ, C. (2009): "La planificación de género en las políticas públicas" en APARICIO, M., et alt. (editoras): Cuadernos de género: políticas y acciones de género. materiales deformación. Madrid: Universidad Complutense de Madrid.

DEERE, C., ALVARADO, G., TWYMAN, J. (2018). ¿Dueñas o jefas del hogar? 
Analizando la desigualdad de género en la propiedad de activos en América Latina. Cuestiones económimcas, vol. 28, pp. 13-40.

DRAVET, F., PASQUIER, F., COLLADO, J., de CASTRO, G. (coord.) (2019). Transdisciplinaridade e Educação do Futuro. Brasilia: Cátedra UNESCO de Juventude, Educação e Sociedade - Universidade Católica de Brasilia.

FREIRE, P. (1971). Pedagogía del oprimido. Montevideo: Ed. San Santiago.

FREIRE, P. (1997). La educación como práctica de la libertad. México DF.: S.XXI ed.

FULLER, N. (1998): "reflexiones sobre el machismo en américa latina", en VALDÉS, T. y OLAVARRÍA, J. (eds.) masculinidades y equidad de género en américa latina. Santiago de Chile: FLACSO.

GARCÍA, E. (2008): políticas de igualdad, equidad y gender mainstreaming ¿de qué estamos hablando?, marco conceptual. El salvador: PNUD.

GIOSA, N. Y RODRÍGUEZ, C. (2010). Estrategias de desarrollo y equidad de género: una propuesta de abordaje y su aplicación al caso de las industrias manufactureras de exportación en méxico y centroamérica. Serie mujer y desarrollo, 97. Santiago: CEPAL.

GRIMALDO, A.; CERVERA, C. (2016). Equidad de género en la educación primaria. Construcciones y deconstrucciones. Jóvenes en la ciencia, vol. 2, n¹, pp. 764768.

HIDALGO, A.; GARCíA, S.; CUBILLO, A.; MEDINA, N. (2019). Los Objetivos del Buen Vivir. Una propuesta alternativa a los Objetivos de Desarrollo Sostenible. Iberoamerican Journal of Development Studies, 8 (1), 6-57.

INSTITUTO INTERNACIONAL DE EDUCACIÓN SUPERIOR EN AMÉRICA LATINA Y EL CARIBE (2020). Covid-19 y educación superior: de los efectos inmediatos al día después. Análisis de impactos, respuestas políticas y recomendaciones. Caracas: IESALC.

KABEER, N. (1999): "the conditions and consequences of choice: reflections on the measurement of womens empowerment", en UNRISD discussion paper 108. IIDH (2000): Convención CEDAW y protocolo facultativo, Costa Rica.

MÁRQUEZ, Y., GUTIÉRREZ, J., GÓMEZ, N. (2017). Equidad, género y diversidad en educación. European Scientific Journal, vol. 13, nº 7, pp. 300-319.

MOMSEN, J. (2019). Gender and Development. New York: Routledge.

NACIONES UNIDAS (2015). Los Objetivos de Desarrollo del Milenio. Informe 2015. New York: ONU.

NUSSBAUM, M. (2000). Women and human development: the 
capabilities approach. Cambridge: Cambridge University Press.

ROCHA, J. y ROCHA, D. (2013). Observatório da Educação Direitos Humanos, Cidadania e Violência: uma estratégia para a articulação da Pós-graduação com a Educação Básica e Formação Continuada dos Profissionais da Educação. In: Global Education Magazine, vol. 6, pp. 49-53.

ROCHA, J. y ROCHA, D. (2014). Mulheres Rurais: Cooperação Internacional para Estudos Multidisciplinares de Gênero, Educação, Cidadania e Responsabilidade Social da Universidade. In: Global Education Magazine, vol. 7, pp. 46-50.

OBSERVATORIO DE IGUALDAD DE GÉNERO DE AMÉRICA LATINA Y ELCARIBE (2008): declaración de parís sobre la eficacia de la ayuda al desarrollo y programa de acción de accra. [en línea]: http://www.americalatinagenera.org/es/index.php?option=0 m_content\&task=view\&id=905\&pub_id=1909

PAHO (2016). Acelerar el progreso hacia la reducción del embarazo en la adolescencia en América Latina y el Caribe. Informe de consulta técnica. Washington DC: PAHO.

SAGREDO, J., \& GALARZA, A. (2020). Economía rural e inequidad de género en el cantón chillanes, provincia de bolívar. Revista De Investigación Enlace Universitario, 19(1), 49-62. https://doi.org/10.33789/enlace.19.1.59

SARAVÍ, G. (2020). Acumulación de desventajas en América Latina: aportes y desafíos para el estudio de la desigualdad. Revista Latinoamericana de Población, vol. 14, $\mathrm{n}^{\circ}$ 27, pp. 228-256. http://doi.org/10.31406/relap2020.v14.112.n27.7

SRINIVASAN, S., RODRíGUEZ, A. (2016). Pobreza y desigualdades rurales. Perspectivas de género, juventud y mercado de trabajo. Serie Desarrollo Productivo. Santiago: CEPAL.

UNESCO (2020). Crisis y currículo durante el COVID-19: mantención de los resultados de calidad en el contexto del aprendizaje remoto. Paris: UNESCO. 